\author{
„IN DER MOLEKULARGENETIK STEHT DER HOFFNUNG, ERBKRANK- \\ HEITEN ZU ERKENNEN UND ZU BEHANDELN, DIE ANGST GEGENÜBER, \\ DIE LETZTE INTIMITÄT ALS INDIVIDUUM ZU VERLIEREN. NUN GILT \\ ES, VERANTWORTUNGSVOLL MIT DEM WISSEN UM DIE EIGENEN \\ GENETISCHEN MÖGLICHKEITEN UND GEFAHREN UMZUGEHEN.“
}

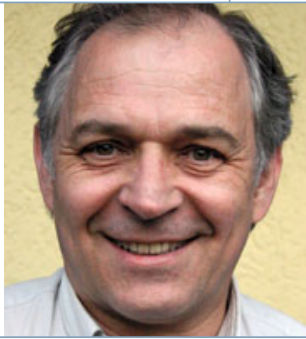

Jochen Graw

\title{
Der Januskopf der genetischen Diagnostik
}

Es wird jetzt ernst: Die genetische Diagnostik für jedermann und jedefrau ist zu haben - zwischen 1.500 und 2.500 Dollar gibt es jetzt die Möglichkeit, die gesamten codierenden Sequenzen eines Menschen sequenzieren zu lassen. Gut, das ist nicht das ganze Genom, sondern nur ein Prozent davon, aber man kann davon ausgehen, dass man 85 Prozent der Krankheitsgene damit fischt. Und das Ganze dauert nur noch sechs Wochen. Für 125 Dollar gibt's dann auch noch die bioinformatische Analyse dazu, die die „gewöhnlichen“ Polymorphismen heraussortiert, sodass man am Ende tatsächlich mit einer Mutationsanalyse dasteht. Nun ist das sicher nicht das Ende der Fahnenstange, und auch die Vollsequenzierung zu einem ähnlich günstigen Preis ist sicher nur noch eine Frage der Zeit. Wenn wir einen Moment innehalten: Es dauerte etwa 15 Jahre, bis das erste gesamte menschliche Genom sequenziert war, und die Welt feierte es mit Spezialausgaben in Nature und Science. Der nächste Schritt war die kommerzielle Analyse von etwa einer Million der bekannten Varianten - auch für etwa 1.000 Dollar. Entwicklungssprünge wie im Rausch - der Preis bleibt gleich, aber die Qualität steigt exponentiell.

Von Anfang an stand die Molekulargenetik unter kritischer Beobachtung - was machen „die Forscher“ da mit „unseren Genen“? Von Anfang an war die Molekulargenetik mit ambivalenten Erwartungen konfrontiert: der Hoffnung, Erbkrankheiten zunächst besser zu erkennen, um sie dann schließlich heilen zu können. Und dem gegenüber stand die Angst, eine letzte Intimität zu verlieren, den Kern der eigenen Individualität. Und von Anfang an stand die Forderung nach einem Moratorium: 1975 auf der Konferenz von Asilomar mündete es in die OECD-

Es gibt viele Gruppen, die an ethischen Fragen zur Gendiagnostik arbeiten - es sei hier nur auf zwei davon hingewiesen:

Die Gendiagnostik-Kommission des Robert-KochInstituts: www.rki.de/DE/Content/Institut/Kommissio nen/GendiagnostikKommission/GEKO_inhalt.html Die Gesellschaft für Humangenetik: http://gfhev.de
Richtlinien zur Gentechnik und in das deutsche Gentechnik-Gesetz. Und aus der Angst vor dem genetisch gläsernen Menschen entstand das Gendiagnostik-Gesetz.

Nun ist es natürlich richtig, dass Krankenversicherungen und auch manch' andere nicht unbedingt wissen müssen, welche Allelvarianten ich habe. Aber - und damit sind wir bei der wirklich wichtigen Frage: Was wollen Eltern wissen, wenn (oder bevor) sie Kinder bekommen?

Natürlich wollen Eltern gesunde Kinder. Das hat nichts damit zu tun, dass man sie sich aus einem Hochglanzprospekt aussucht. Sondern einfach damit, dass Eltern ihren Kindern Leiden ersparen möchten und sich selbst die tagtäglichen Sorgen um die Gesundheit der Kinder. Wer selbst behinderte Kinder hat, kann das sicher verstehen - einerseits. Andererseits: Auch kranke Kinder haben nur dieses eine Leben, und sie kennen es nicht anders. Sind sie unglücklich?

In der heutigen Diskussion um Gendiagnostik ist häufig von „Selektion“ die Rede. Das ist vor der deutschen Geschichte verständlich - das Bild von der Rampe in Auschwitz steht jedem unmittelbar vor Augen und jene Ärzte, die mit „lebensunwertem Leben“ und mit „unnormalen Kindern“ ihre Experimente machten. Einerseits. Und andererseits: Wie überfordert sind Eltern, wenn sie ein Leben lang ihr Kind pflegen müssen, auch in dem Bewusstsein, dass sie es am Ende ihres eigenen Lebens nicht mehr tun können, und wer soll es dann tun? Sicher, Ähnliches kann auch nach einem Unfall passieren - aber von Anfang an? Und vielleicht in dem Bewusstsein, man hätte es vermeiden können, wenn ...? Heute stehen junge Menschen, die Eltern werden wollen, in einer historisch einmaligen Situation: Sie können erfahren, welche Anlagen für Krankheiten in ihren Genen schlummern - wenigstens in Ansätzen, denn alles ist ja immer noch nicht bekannt. Dafür gibt es nichts Vergleichbares in früherer Zeit, an dem man sich orientieren könnte - höchstens die Geschichte mit dem Apfel vom Baum der Erkenntnis - und dem Genuss dieses „Apfels“ folgte die Vertreibung aus dem Paradies, aus dem Paradies der Unkenntnis, des Nicht-Wissens, aber auch aus dem Paradies, selbst keine Verantwortung übernehmen zu müssen, sondern dies dem lieben Gott überlassen zu können. Mit der Möglichkeit, genetische Informationen zu erhalten oder genau diese Information ausschlagen zu können, werden wir zu Entscheidungen gezwungen, denen wir uns eigentlich lieber nicht stellen möchten. Die Frage „was wäre, wenn“ wird sich Eltern zukünftig immer stellen, wenn es um Gesundheit und Krankheit ihrer Kinder geht, und natürlich auch um ihre eigene Gesundheit und Krankheit.

Durch gesetzliche Gebote oder Verbote werden wir nicht sehr weit kommen. Denn „einmal Gedachtes oder Entdecktes kann nicht rückgängig gemacht werden“, wie es in Dürrenmatts Die Physiker heißt. Es bleibt nur die Macht der Aufklärung, nach manchen Irrwegen einen verantwortungsvollen Weg zu finden, mit dem Wissen um die eigenen genetischen Möglichkeiten und Gefahren umzugehen.
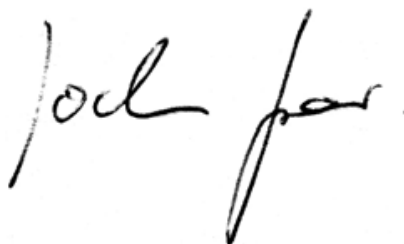

Jochen Graw

Helmholtz Zentrum München

Korrespondenzadresse:

Prof. Dr. Jochen Graw

Helmholtz Zentrum München

Deutsches Forschungszentrum für Gesundheit und Umwelt GmbH

Institut für Entwicklungsgenetik, Arbeitsgruppe Augenerkrankungen Ingolstädter Landstraße 1

D-85764 Neuherberg

Tel.: 089-3187-2610

Fax: 089-3187-4620

graw@helmholtz-muenchen.de

www.helmholtz-muenchen.de 